\title{
Adaptive Decision Feedback Orthogonality Restoration Filter for Windowed OFDM
}

\author{
Pinchieh Huang Yumin Lee \\ Dept. of Electrical Engineering and \\ Graduate Institute of Communication Eng., \\ National Taiwan University, Taipei 10617, Taiwan \\ yuminlee@cc.ee.ntu.edu.tw
}

\begin{abstract}
OFDM is a promising multi-carrier modulation technique that can effectively mitigate impairments caused by wireless communication channels. In some OFDM standards (e.g., IEEE 802.11a), windowing is employed to reduce sensitivity to frequency offset and phase noise, and at the same time conserve bandwidth. However, windowing causes the loss of orthogonality between subearriers. In this paper, we proposed a decision feedback orthogonality restoration filter for minimizing orthognality loss due to both windowing and inter-block interference. Simulation results using IEEE 802.11a parameters show that the proposed scheme achieves significant performance improvement.
\end{abstract}

\section{INTRODUCTION}

The demand for high data rate services in the wireless environment is rapidly increasing due to the spectacular growth of voice and video communications over the Internet. A plethora of research and development take place around the world to define the next generation wireless multimedia communications system. One key issue in these systems is finding a suitable modulation scheme to combat the wireless channel impairments. Orthogonal frequency division multiplexing (OFDM) is one such modulation that has been adopted by both IEEE 802.11[1] and European Telecommunication Standards Institute Broadband Radio Access Network (ETSI BRAN)[2]. Both IEEE 802.11a[3] and the ETSI High Performance Local Area Network type 2 (HIPERLAN/2) target a range of data rates from 6 up to 54 Mbps in the $5 \mathrm{GHz}$ band, which makes OFDM effectively a worldwide standard for broadband wireless communications. Furthermore, OFDM is also being considered for future high-speed wireless data network such as wireless local loop (WLL), and the so-called "fourth-generation" systems.

It is well known that OFDM is very sensitive to frequency offset and phase noise that cause inter-carrier interference (ICI). One possible method for mitigating this sensitivity is to introduce time-domain windowing at the OFDM transmitter. Windowing reduces the sidelobes of the OFDM signal spectrum, thus reducing the sensitivity to frequency offset and conserving bandwidth. However, as shown in this paper, for windowed OFDM the subcarriers lose orthogonality after the cyclic prefix $(\mathrm{CP})$ is removed at the receiver, even if the memory of the channel is within the length of CP. The loss of orthogonality due to time-domain windowing is a time-varying phenomenon even when the channel is stationary, and results in a bit error rate (BER) floor that can be reduced only by using special receiver signal processing techniques. If the memory of the channel exceeds the length of $\mathrm{CP}$, significant performance degradation results due to inter-block interference (IBI) regardless of whether time-domain windowing is used.

In this paper we propose an algorithm for windowed OFDM receivers referred to as the decision feedback orthogonality restoration filter (DFORF). In the proposed scheme, a feedback filter is used to reduce the IBI caused by severe multipath, and a feedforward filter is used for restoring orthogonality between subcarriers. The DFORF requires an estimate of the time-domain discrete-time equivalent channel. In this paper, the recursive least squares (RLS) algorithm[4] and interative channel interpolation[5] are used to track the channel frequency response, and the time-domain discrete-time equivalent channel is obtained by taking the inverse discrete Fourier transform (IDFT) of the estimated channel frequency response. Simulation shows that DFORF greatly enhances the multipath mitigating capability of windowed OFDM and can replace the role of the equalizer in a conventional OFDM receiver.

\section{SYSTEM MODEL}

The system model used throughout this paper is shown in Figure 1. Let $x(k, m), m=0,1, \ldots, N-1$, be the time-domain samples of the $k$-th transmitted OFDM symbol (excluding CP), where $N$ is the number of subcarriers (size of FFT). The CP is prepended to $x(k, m)$ and the resulting signal is multiplied by a time-domain window function given by $w(m), m=0,1, . ., N_{g}-1, N_{g}, \ldots . N_{g}+N-1$, where $N_{g}$ is the length of CP. The total number of samples in an OFDM symbol is thus $N_{T}=N+N_{g}$. The resulting signal is given by

$$
x^{\prime}(m)=\sum_{k=0}^{K-1} x\left(k,\left(m-k N_{T}-N_{g}\right)_{N}\right) w(m)
$$

where $(\bullet)_{N}$ is the modulo- $N$ operator and $K$ is the total number of OFDM symbols. This signal is transmitted through the wireless channel, which is modeled as a multipath fading channel corrupted by additive white Gaussian noise (AWGN). The discrete-time received signal is given by 


$$
y^{\prime}(m)=\sum_{j=0}^{v} p_{j} x^{\prime}(m-j)+n(m)
$$

where $p_{j}, j=0,1, \ldots, \mathrm{v}$, is the time-domain discrete-time equivalent channel and $n(m)$ are the AWGN samples. At the receiver, $\mathrm{CP}$ is discarded and the resulting signal is given by

$$
y(k, m)=y^{\prime}\left(k N_{T}+N_{g}+m\right)
$$

for $m=0,1, \ldots, N-1$ and $k=0,1, \ldots, K-1$. The signal $y(k, m)$ is next passed through a serial-to-parallel $(\mathrm{S} / \mathrm{P})$ converter and subsequently processed by the proposed DFORF shown in Figure 1. DFT is performed on the output of the DFORF, and the result is optionally processes by a frequency-domain equalizer and slicer to obtain the final output.

\section{DECISION FEEDBACK ORTHOGONALITY RESTORATION FILTER}

In this paper, we only consider this case where $N_{g}<v \leq$ $N_{T}$, although the proposed scheme can be easily extended to the general case. Let

$$
\mathbf{X}(k) \equiv\left[\begin{array}{lll}
x(k, 0) & \cdots & x(k, N-1)
\end{array}\right]^{\mathrm{T}}
$$

and

$$
\mathbf{Y}(k) \equiv\left[\begin{array}{lll}
y(k, 0) & \cdots & y(k, N-1)
\end{array}\right]^{\mathrm{T}},
$$

where " $T$ " denotes matrix transposition. We can easily show that

$$
\mathbf{Y}(k)=\mathbf{P}_{1} \mathbf{W}_{1} \mathbf{X}(k)+\left[\begin{array}{cc}
\mathbf{0} & \mathbf{P}_{2} \mathbf{W}_{2} \\
\mathbf{0} & \mathbf{0}
\end{array}\right] \mathbf{X}(k-1)+\mathbf{N}(k),
$$

where $\mathbf{P}_{1}$ is an $N \times N_{T}$ circulant matrix given by

$$
\mathbf{P}_{1} \equiv\left[\begin{array}{cccccccccc}
p_{0} & 0 & \cdots & \cdots & \cdots & 0 & p_{v} & \cdots & \cdots & p_{1} \\
p_{1} & p_{0} & 0 & \cdots & \cdots & \cdots & 0 & p_{v} & \cdots & p_{2} \\
\vdots & \ddots & \ddots & \ddots & \ddots & \ddots & \ddots & \ddots & \ddots & \vdots \\
0 & \cdots & 0 & p_{v} & \cdots & p_{0} & 0 & \cdots & \cdots & 0
\end{array}\right]
$$

and $\mathbf{W}_{1}$ is an $N_{T} \times N$ matrix that is given by

$$
\mathbf{W}_{1} \equiv\left[\begin{array}{cc}
\mathbf{A} \\
\mathbf{O} & \mathbf{B}
\end{array}\right] \text {, }
$$

where $\mathbf{A}=\operatorname{diag}\left(w\left(N_{g}\right), w\left(N_{g}+1\right), \ldots . w\left(N_{T}-1\right)\right)$ and $\mathbf{B}=$ $\operatorname{diag}\left(w(0), w(1), \ldots w\left(N_{g}-1\right)\right), \mathbf{P}_{2}$ is a $\left(v-N_{g}\right) \times\left(v-N_{g}\right)$ Toeplitz matrix given by

$$
\mathbf{P}_{2}=\left[\begin{array}{cccc}
p_{v} & p_{v-1} & \cdots & p_{N_{g}+1} \\
0 & p_{v} & \cdots & p_{N_{g}+2} \\
\vdots & \ddots & \ddots & \ddots \\
0 & \cdots & 0 & p_{v}
\end{array}\right]
$$

$\mathbf{W}_{2}=\operatorname{diag}\left(\mathrm{w}\left(N_{T}+N_{\mathrm{g}}-\mathrm{v}\right), \mathrm{w}\left(N_{T}+N_{\mathrm{g}}-\mathrm{v}+1\right), \ldots \mathrm{w}\left(N_{T}-1\right)\right)$, and $\mathbf{N}(k)$ is an $N \times 1$ vector of AWGN samples. The first and second terms in (4) characterize, respectively, the combined effect of the channel and time-domain windowing and IBI from the previous OFDM symbol resulting from the assumption $N_{g}<v$.

The proposed DFORF consists of a feedforward filter and feedback filter, as shown in Figure 1. The feedback filter is used to synthesize the IBI from the estimate of the previous OFDM symbol, which is in turn subtracted from $\mathbf{Y}(k)$. The resulting IBI-free received OFDM symbol is given by

$$
\mathbf{Y}^{\prime}(k)=\mathbf{Y}(k)-\left[\begin{array}{cc}
\mathbf{0} & \mathbf{P}_{2} \mathbf{W}_{2} \\
\mathbf{0} & \mathbf{0}
\end{array}\right] \hat{\mathbf{X}}(k-1) .
$$

It should be noted that if $N_{g} \geq v$, there is no IBI and (8) reduces to $\mathbf{Y}(k)=\mathbf{Y}(k)$ as one would expect. In the DFORF, $\mathbf{Y}^{\prime}(k)$ is subsequently processed by the feedforward filter $\mathbf{L}$. The feedforward filter $\mathbf{L}$ is obtained based on the minimum mean square error (MMSE) criterion [6], i.e., $\mathbf{L}$ is chosen such that

$$
\mathbf{K}=E\left[\left(\mathbf{X}(k)-\mathbf{L} \mathbf{Y}^{\prime}(k)\right)\left(\mathbf{X}(k)-\mathbf{L} \mathbf{Y}^{\prime}(k)\right)^{H}\right]
$$

has the property that $\mathbf{c}^{H} \mathbf{K c}$ is minimum for any vector $\mathbf{c}$. The output of the DFORF is given by

$$
\hat{\mathbf{X}}(k)=\mathbf{W}_{1}{ }^{H} \mathbf{P}_{1}\left[\mathbf{P}_{1} \mathbf{W}_{1} \mathbf{W}_{1}{ }^{H} \mathbf{P}_{1}{ }^{H}+\frac{N}{E_{S}} \mathbf{R}_{N}\right]^{-1} \mathbf{Y}^{\prime}(k)
$$

where " $\mathrm{H}$ " denotes Hermitian transposition, $\mathbf{R}_{N}$ is the autocorrelation matrix of $\mathbf{N}(k)$, and $E_{S}$ is the energy of each OFDM subsymbol. The output of the feedforward filter is the MMSE estimate of the transmitted $k$-th OFDM symbol $\mathbf{X}(k)$, and is used by the subsequent FFT and equalization processes to yield the recovered user data, as shown in Figure 1. From (10), we can see that the DFORF cancels the adverse effects of the channel, windowing and noise, so it can minimize the performance degradation, which will be shown later. Furthermore, if the channel estimate is sufficiently accurate, the DFORF can completely replace the subsequent equalizer, which is also shown later in the simulation results. Finally, if $v \leq N_{g}$ then $\mathbf{P}_{2}=\mathbf{0}$, and the feedback filter is turned off. The resulting feedforward-only filter is referred to as the orthogonality restoration filter (ORF). Note that the output of the ORF is obtained by replace $\mathbf{Y}^{\prime}(k)$ with $\mathbf{Y}(k)$ in (10) 


\section{CHANNEl ESTIMATION, INTERPOLATION AND POST-PROCESSING}

It is clear from the previous section that the DFORF requires an estimate of the time-domain discrete-time equivalent channel, which can be obtained by taking the IDFT of the estimated frequency-domain channel response. In this paper, we follow IEEE 802.11a specifications and assume that training symbols are available for synchronization purposes. These training symbols are also used for obtaining an initial estimate of the channel frequency response. Furthermore, in order to make channel estimation more accurate, we also adopt the RLS algorithm so that the channel estimates are refined on a decision-aided basis.

In IEEE $802.11 \mathrm{a}$, the training symbols do not use all subcarriers. Only 52 out of the 64 subcarriers are used for carrying training symbols. The 12 unused subcarriers serve as guard band for implementation purpose. The channel gains on these unused subcarrier frequencies obviously cannot be estimated directly, and must be interpolated from the estimated channel gains of the active subcarriers. However, ideal sinc interpolation[7] is not possible here because the unused subcarriers are contiguous. In this paper, we adopt an iterative scheme[5] for interpolating the channel gain estimates of the unused subcarrier frequencies. After the complete channel frequency response is obtained, taking the IDFT of the channel frequency response yields the time-domain discrete-time equivalent channel impulse response that is required by the DFORF.

Based on the above discussions, one would expect that the DFORF can effectively restore subcarrier orthogonality and remove the adverse effects of IBI provided that the channel estimates are perfect, therefore the frequency-domain equalizer in Figure 1 is no longer necessary. However, since the time-domain discrete-time equivalent channel impulse response is obtained using channel estimation and interpolation and is thus inaccurate, a frequency-domain equalizer may still be necessary as a post-processing unit to undo any remaining effects of the channel. If this is the case, then the channel must be re-estimated in order to obtain the "effective" channel gains seen at the output of the DFORF. In this paper, we use the decision-directed RLS algorithm to estimate the effective channel. The estimated effective channel is then used to compute the coefficients for the frequency-domain equalizer. The structure of the frequency-domain equalizer is the same as the equalizer in the conventional OFDM receiver. The necessity and effectiveness of this post-processing remain to be evaluated by simulation.

\section{SimUlation RESULTS}

The performance of the proposed DFORF is simulated using IEEE $802.11 \mathrm{a}$ specifications. The number of subcarriers $N$ is set to 64 , and each active subcarrier is modulated using quaternary phase shift keying (QPSK). As mentioned earlier, 52 of the 64 subcarriers are active while 12 are unused. The length $N_{g}$ of the CP is equal to 16. The window $w(n)$ is a raised-cosine function. Preamble OFDM symbols are transmitted at the beginning of each burst as specified in the IEEE 802.11a standard. The duration of each OFDM symbol (including CP) is $4 \mu \mathrm{s}$. The wireless channel is simulated using the Jakes fading model[8] with exponential power-delay profile with various values of root-mean-square (RMS) delay-spread (DS) and maximum Doppler spread. The carrier frequency is equal to $5 \mathrm{GHz}$. No frequency offset is assumed in the simulations.

The BER of the proposed method is shown in Figure 2 as functions of $E_{b} / N_{o}$ for DS of $78 \mathrm{~ns}$ and $355 \mathrm{~ns}$, where $E_{b}$ is the transmitted energy per bit and $N_{0} / 2$ is the two-sided AWGN power spectral density. The maximum Doppler shift frequency, $f_{m}$, is $18.52 \mathrm{~Hz}$ (corresponding to a walking speed of $4 \mathrm{~km} /$ hour). The BER of a conventional OFDM receiver is also shown as baselines for comparison. A BER floor can be observed in Figure 2 for the conventional receiver for both values of DS. The proposed method with DFORF successfully removes the BER floor for DS of 78ns, but for DS of 355 ns the BER floor is only reduced slightly when the ORF is used. This is because for DS of $78 \mathrm{~ns}$ the BER is mainly due to windowing, while for DS of $355 \mathrm{~ns}$ the floor is due to both windowing and IBI. As can be seen in Figure 2, DFORF completely removes the BER floor for DS of $355 \mathrm{~ns}$ because it eliminates the effect of both IBI and windowing.

The BER of different receivers are also shown in Figure 3 as functions of DS ( $E_{b} / N_{0}$ is fixed at $\left.25 \mathrm{~dB}\right)$. Here, we use the "perfect" channel estimation. For the conventional receiver and the proposed receiver with the ORF, the BER begins to increase when DS increases beyond 200 ns (about $25 \%$ of CP). This is due to IBI caused by the large DS. However, the BER with the conventional receiver is still larger than that with the ORF because of orthogonality loss due to windowing. On the other hand, for DFORF the BER remains almost constant even when DS is increased to $400 \mathrm{~ns}$. Therefore the DFORF in the proposed scheme effectively doubles the tolerable DS (to about $50 \%$ of CP). Finally, for ideal decision feedback, a noiseless copy of the previous OFDM symbol is used as the input of the feedback filter. Since this is not realizable, the resulting BER serves only as a baseline for comparison. As can be seen in Figure 3, for $E_{b} / N_{0}$ of $25 \mathrm{~dB}$, the error propagation associated with DFORF results only in a very slight loss of performance when DS is greater than $400 \mathrm{~ns}$.

Figure 4 shows the BER of the proposed receiver with the ORF and DFORF with and without using the frequency-domain equalizer as a post-processor. The maximum Doppler shift frequency $f_{m}$ is $18.52 \mathrm{~Hz}$ (corresponding to a walking speed of $4 \mathrm{~km} / \mathrm{hour}$ ). It can be seen that the performance is slightly better without the 
frequency-domain equalizer. This is because at a relatively low Doppler frequency shift, the channel estimate is reasonably accurate. The frequency-domain equalizer in this case only adds unnecessary noise into the receiver, which makes the performance slightly worse. In other words, when Doppler is small the ORF or DFORF can replace the frequency-domain equalizer in a conventional OFDM receiver. We also note that both curves saturate due to Doppler effect.

The BER of ORF with and without frequency-domain equalizer is shown in Figure 5 as functions of relative speed between the transmitter and receiver. The RMS DS is $78 \mathrm{~ns}$ and $E_{b} / N_{0}$ is fixed at $35 \mathrm{~dB}$. It can be seen that the performance without frequency-domain equalizer is better when the relative speed is slower than $55 \mathrm{~km} / \mathrm{hr}$ (i.e., $f_{m}$ $=254.63 \mathrm{~Hz}$ ). On the other hand, it is better to have a frequency-domain equalizer as a post-processor only when the relative speed is over $55 \mathrm{~km} / \mathrm{hr}$; and even in that case the performance gain due to the post-processor is very small Since IEEE 802.11a is used in indoor environments with very low Doppler frequencies, the frequency-domain equalizer unnecessary. Similar trends are observed for the DFORF at higher values of DS.

Figure 6 shows simulation results for windowed OFDM using DFORF and OFDM without windowing using the conventional receiver. The maximum Doppler shift frequency, $f_{m}$, is $18.52 \mathrm{~Hz}$ (corresponding to a walking speed of $4 \mathrm{~km} /$ hour). It can be seen that windowing with DFORF receiver achieves better performance than that without windowing using the conventional receiver, especially when $E_{b} / N_{0}$ is over $34 \mathrm{~dB}$. This is because windowing reduces the impact of Doppler spread, provided that the DFORF is used at the receiver to restore orthogoanlity between subcarriers. In other words, when windowing is used in conjunction with DFORF, OFDM performance benefits from the advantages of reduced sensitivity to Doppler effect without suffering too much from the side effect of loss of subcarrier orthogonality.

\section{CONCLUSION}

A decision-feedback orthogonality restoration filter (DFORF) is proposed for windowed OFDM systems. Simulations using the IEEE 802.1 la specifications show that the DFORF can minimize the loss of subcarrier orthogonality due to windowing and inter-block interference (IBI), thus significantly improving performance. Furthermore, although the proposed DFORF requires time-domain channel estimates, for IEEE 802.11a in an indoor environment with small Doppler spread the time-domain channel can be sufficiently estimated from training symbols, such that frequency-domain equalization is no longer necessary. By simulation we have demonstrated that DFORF enables the OFDM system to benefit from the advantages of windowing without suffering from its side effects. Although the simulation results are for IEEE 802.11 a only, we believe that the conclusions can be extended to the general case.

\section{REFERENCES}

[1] IEEE 802.11 Website URL: http:/grouper.ieee.org/groups/802/11

[2] ETSI Website URL: http://www.etsi.org

[3] IEEE, "Supplement To The Standard for Telecommunications and Information Exchange Between Systems - LAN/MAN Specific Requirements - Part 11: Wireless MAC and PHY Specifications: High-Speed Physical Layer in the 5-GHz Band," IEEE 802.11a-1999.

[4] Simon. Haykin, Adaptive filter theory, $3^{\text {rd }}$ Ed., Prentice-Hall, 1996

[5] Yumin Lee and Pinchieh Huang, "Channel interpolation and MMSE multi-input multi-output frequency-domain DFE for wireless data communications using OFDM," submitted to 2001 IEEE Global Telecommunications Conference.

[6] Simon Haykin, Modern Filters, Macmillan Publishing Company, 1989.

[7] L. Hanzo, W. Webb, and T. Keller, Single-And Multi-Carrier Quadrature Amplitude Modulation, Wiley, 2001.

[8] P. Dent, G. E. Bottomley, and T. Croft, "Jakes' Fading Model Revisited," Electronics Letters, Vol. 29 No. 13, 24 June 1993.

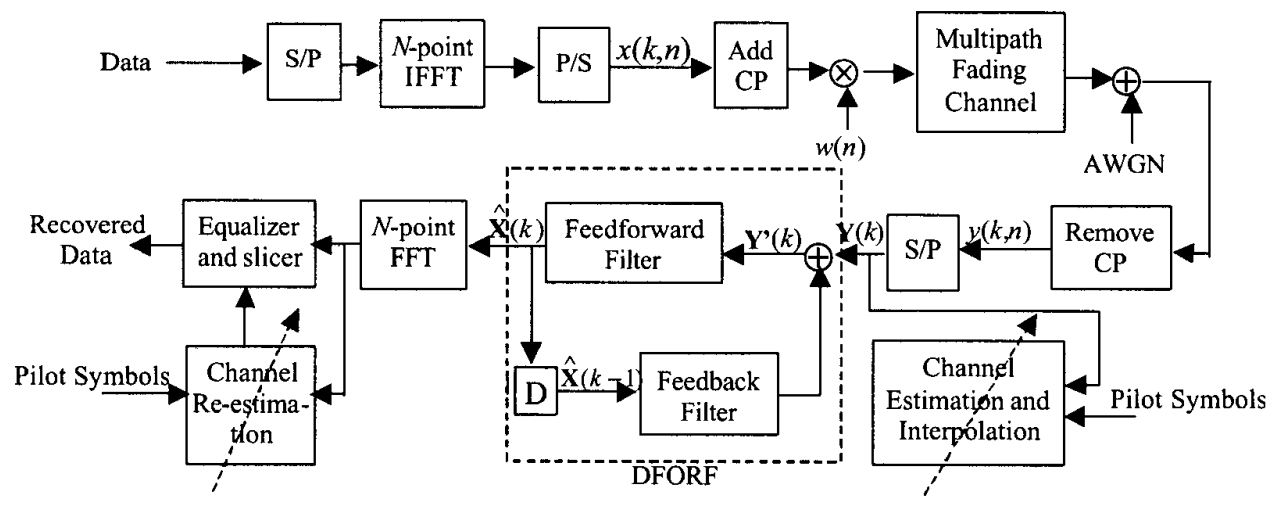

Fig. 1. A windowed OFDM transmission system with the proposed DFORF. 


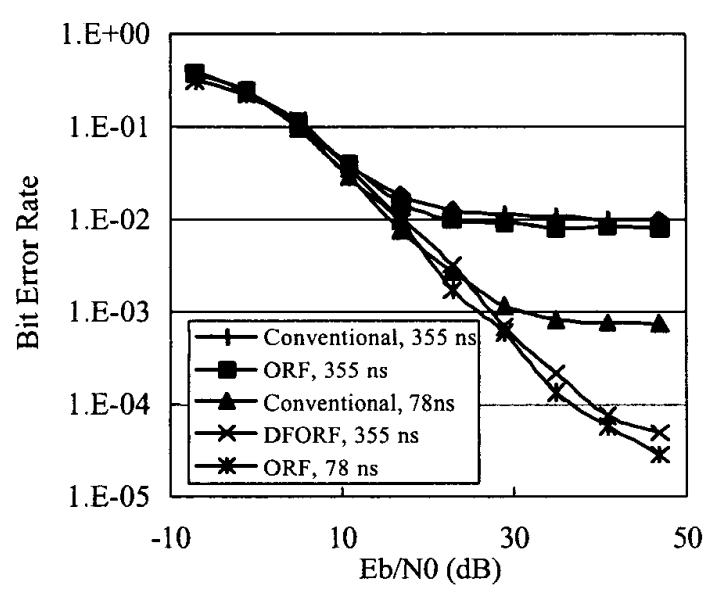

Fig. 2. BER of various receivers as functions of $E_{b} / N_{0}$.

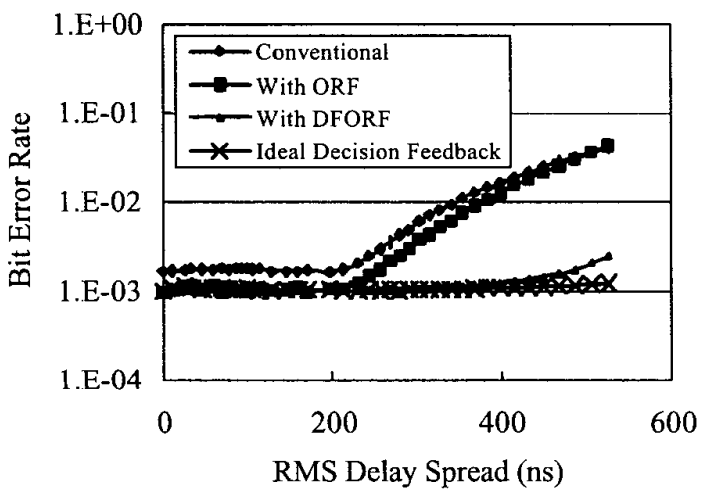

Fig. 3. BER of various receivers as functions of the RMS delay spread. $E_{b} / N_{0}$ is fixed at $25 \mathrm{~dB}$.

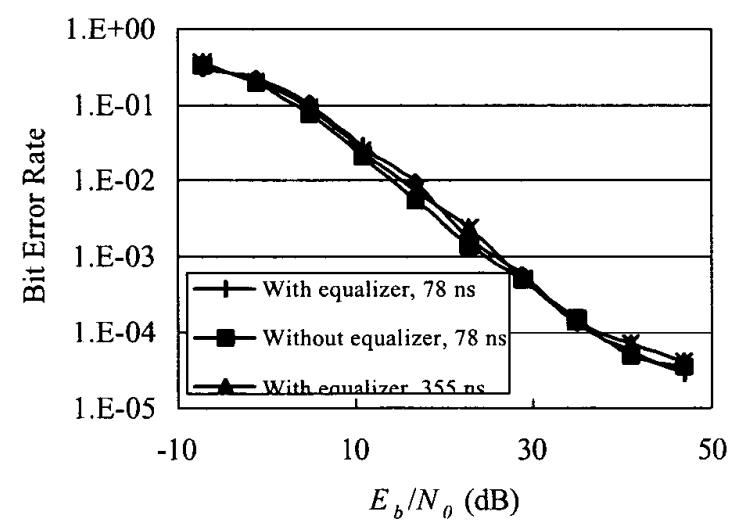

Fig. 4. BER of DFORF with and without equalizer as functions of $E_{b} / N_{0}$.

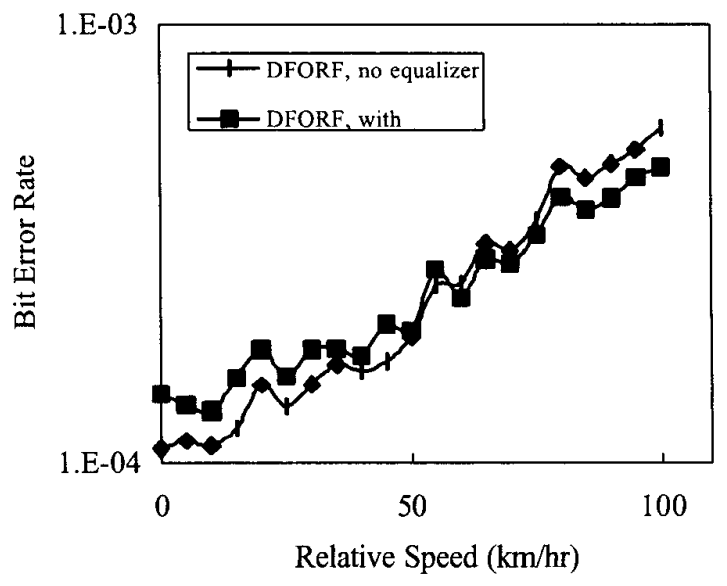

Fig. 5. BER of DFORF with and without equalizer as functions of relative speed. RMS delay-spread is $78 \mathrm{~ns}$ and $E_{b} / N_{0}$ is $35 \mathrm{~dB}$.

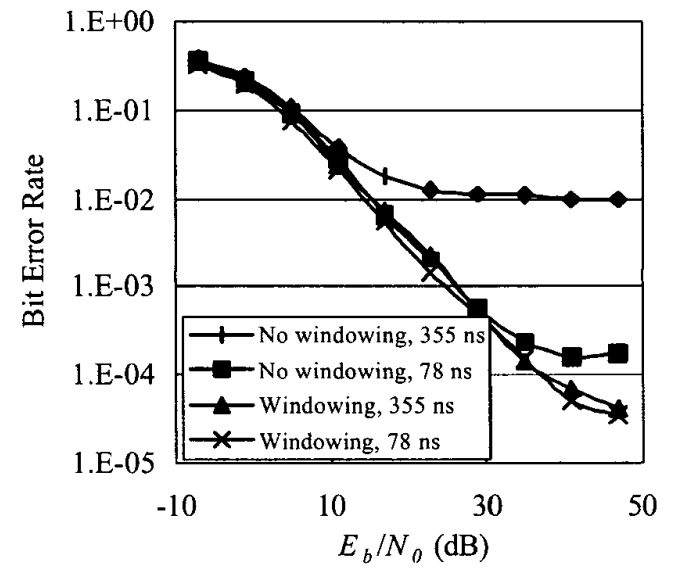

Fig. 6. BER of OFDM with and without windowing as functions as $E_{b} / N_{0}$. DFORF is used with windowing, and the conventional receiver is used for the case without windowing. 